\title{
Distinctive gut microbial dysbiosis between chronic alcoholic fatty liver disease and metabolic-associated fatty liver disease in mice
}

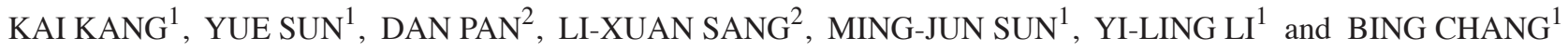 \\ Departments of ${ }^{1}$ Gastroenterology and ${ }^{2}$ Geriatrics, The First Affiliated Hospital, \\ China Medical University, Shenyang, Liaoning 110001, P.R. China
}

Received May 25, 2020; Accepted January 18, 2021

DOI: $10.3892 /$ etm.2021.9862

\begin{abstract}
The gut microbiota, which may affect normal physiological and biochemical functions, has an important role in the development of human liver diseases. The aim of the present study was to investigate differences in the gut microbiota between chronic alcoholic fatty liver disease (AFLD) and metabolic-associated fatty liver disease (MAFLD). AFLD was induced by chronic alcohol administration and MAFLD was induced by a Western-style diet in C57BL/6 mice. After 8 weeks, the levels of plasma alanine aminotransferase (ALT), aspartate aminotransferase (AST), triglyceride (TG), total cholesterol (TC), lipopolysaccharide (LPS), tumor necrosis factor (TNF)- $\alpha$, interleukin (IL)-6, IL-1 $\beta$ and IL-10 were assessed and H\&E staining of mouse liver tissue was performed. High-throughput sequencing of $16 \mathrm{~S}$ ribosomal DNA from the intestinal contents was used to analyze the different effects of AFLD and MAFLD on the gut microbiota. Differences in the gut microbiota composition were assessed by the t-test. The results revealed increases in LPS, ALT, AST, TG, IL-1 $\beta$ and TNF- $\alpha$ in the AFLD group. Compared with those in the MAFLD control group, the MAFLD group exhibited increased plasma ALT, TG, TC, IL-6, IL-1 $\beta$ and TNF- $\alpha$ levels and decreased plasma IL-10 levels. In addition, the $\alpha$ - and $\beta$-diversities revealed that the AFLD and MAFLD groups exhibited obvious changes in the gut structure (with an increase in abundance in the AFLD group and a decrease in abundance in the MAFLD group). In comparison to the AFLD control group, Enterococcaceae were the most abundant bacteria at the family level and Enterococcus and Streptococcus were the most abundant bacteria at the genus level in the AFLD group. However, in the MAFLD group,
\end{abstract}

Correspondence to: Professor Yi-Ling Li or Professor Bing Chang, Department of Gastroenterology, The First Affiliated Hospital, China Medical University, 155 Nanjing North Street, Shenyang, Liaoning 110001, P.R. China

E-mail: 1yl-72@163.com

E-mail:cb000216@163.com

Key words: alcohol fatty liver disease, metabolic-associated fatty liver disease, gut microbiota, dysbiosis
Lachnospiraceae was the most abundant at the family level, with increases in Erysipelatoclostridium, Gordonibacter and Streptococcus at the genus level and a decrease in the genus Bifidobacterium. In conclusion, the present study confirmed that the AFLD and MAFLD groups harbored differences in the gut microbiota. The marked differences in the gut microbiota at the family and genus levels may contribute to the development process of FLD.

\section{Introduction}

Fatty liver disease (FLD), which is a major cause of chronic liver disease, is a leading cause of morbidity and mortality worldwide. Chronic alcoholic FLD (AFLD) and metabolic-associated FLD (MAFLD) represent the major forms of FLD and may develop into alcoholic steatohepatitis and metabolic-associated steatohepatitis (MASH), respectively $(1,2)$. Most current estimates suggest that alcohol accounts for up to $50 \%$ of liver cirrhosis-associated deaths among the 2 million patients worldwide who die from liver disease per year, and the worldwide prevalence of MAFLD is $25 \%$ of the general population $(1,3)$. The pathogenesis of AFLD has remained to be fully elucidated. Current studies suggest that it is related to the toxic effects of alcohol and its metabolites in the liver, oxidative stress and the increased release of proinflammatory cytokines mediated by the immune response (4). MAFLD is a complex multifactorial disease that involves a variety of genetic, metabolic and environmental factors and is closely related to various conditions, including insulin resistance, metabolic syndrome, obesity and diabetes (5). Without effective treatment methods, the prognosis and global burden of FLD are not optimistic. Thus, there is an urgent requirement to investigate the pathogenesis of and novel treatment strategies for FLD.

The gut microbiota is a complex microbial environment where dynamic mutualistic interactions related to digestion and the absorption of dietary components take place. The consumption of specific food ingredients may modulate the gut microbiota composition and produce bacterial metabolites with effects on host health (6). After birth, the immune system matures via interactions with microbes in the gut and the interaction between the host and microbiota is considered to be fundamental for the development of the immune system $(7,8)$. Observational findings during the past two decades suggest 
that the gut microbiota may contribute to the metabolic health of the human host and, when aberrant, to the pathogenesis of various common metabolic disorders, including obesity, type 2 diabetes, nonalcoholic liver disease, cardiometabolic diseases and malnutrition (9). Recently, the important role of the gut microbiota and microbial products in the pathogenesis of modulating liver diseases, such as alcohol-associated liver disease, MAFLD, steatohepatitis and cholestatic liver diseases, has become evident (10). A decrease in the relative abundance of Akkermansia and increase in the relative abundance of Veillonella was observed in patients with alcoholic hepatitis with more severe disease, along with a reduction in the Shannon diversity index in antibiotic-treated patients and patients receiving steroids, indicating that the gut microbiota may be an attractive target to prevent and treat alcoholic hepatitis (11). The treatment of mice with fecal microbiota transplant from alcohol-resistant donor mice may prevent alcohol-induced liver injury (12). A previous study suggested that microbiota dysbiosis is the first response of the organism to high-density energy diets, followed by increased liver fat accumulation, microglia activation in the brain and circulating levels of inflammatory markers (13). It has been reported that the abundance of Lachnospiraceae in the fecal samples of patients with MAFLD is significantly higher than that in healthy subjects (14). Kim et al (15) also reported a high abundance of the Lachnospiraceae family in a group of patients with persistent MAFLD compared with their control group. Probiotics, which are defined as 'live microorganisms, which, when administered in adequate amounts, confer a health benefit on the host', have been reported to also benefit patients with MAFLD (16,17). Furthermore, probiotics were reported to improve liver enzymes in patients with alcohol-induced liver injury by improving the gut microbiota (18). All of these studies have demonstrated that the gut microbiota has an important role in the development of FLD. However, to the best of our knowledge, there has been no research comparing the differences in the gut microbiota in AFLD and MAFLD by using simultaneous experiments.

In previous studies, extensive research was performed using animal models of FLD. Different models (Lieber-DeCarli liquid diet, ethanol ad libitum feeding, the Tsukamoto-French model and the model of chronic and binge ethanol feeding (the NIAAA model) employing rodents, which mainly include mice and rats, have been established to investigate the effects of acute and chronic alcohol exposure on the initiation and progression of AFLD (19). To elucidate the pathophysiology of MAFLD, a myriad of different rodent models (dietary, genetic, and chemical rodent models) has been developed and the method of building dietary models is considered to be relatively simple and convenient (20). To date, the C57 family of inbred mouse strains has been identified as having the highest innate ethanol consumption (21). Furthermore, mice of the strains NZW/LacJ, C57BL/10J, FVB/NJ and BALB/cByJ exhibited severe liver injury compared with mice of the WSB/EiJ, $\mathrm{PWD} / \mathrm{PHJ}, \mathrm{C} 3 \mathrm{H} / \mathrm{HeJ}$ and $\mathrm{AKR} / \mathrm{J}$ strains in another study. These results indicated that the marked difference in sensitivity to alcohol was dependent on the mouse strain $(19,22)$. Taking all of the above into consideration, C57BL/6 mice were selected for the present study to investigate differences between AFLD and MAFLD. In the present study, serological markers and the composition of the gut microbiota were assessed to evaluate the different effects of chronic alcohol feeding and a Western-style diet on mice. Understanding the differences in gut microbiota dysbiosis at different levels between AFLD and MAFLD may aid to elucidate the different pathogenetic mechanisms of AFLD and MAFLD, and provide a basis for future research in this field.

\section{Materials and methods}

Animals. A total of 28 male C57BL/6 mice (age, 8 weeks; body weight, $20 \pm 2 \mathrm{~g}$ ) were obtained from Changsheng Laboratory Animal Technology Co., Ltd. Mice were housed in specific-pathogen-free facilities (temperature $23 \pm 2{ }^{\circ} \mathrm{C}$; humidity, 55 \pm \%; 12-h light/dark lighting regimen). Prior to the experiment, the animals were allowed to adapt to the environment for one week. For this study, all procedures were performed in strict accordance with the National Institutes of Health guidelines and were approved by the Animal Research Committee of China Medical University (Shenyang, China; no. 2019061).

Experimental design and induction of chronic AFLD and $M A F L D$. The experimental mice were randomly divided into four groups (n=7/group): The AFLD group, the AFLD control group, the MAFLD group and the MAFLD control group. To induce alcoholic fatty liver, the Lieber-DeCarli liquid diet was used to induce the AFLD mouse model. The AFLD group was fed a modified Lieber-DeCarli liquid diet (35\% fat, $18 \%$ protein and $11 \%$ carbohydrates, and the alcohol calorie intake was 36\%; provided by Trophic Animal Feed High-tech Co., Ltd.) for 8 weeks. The AFLD control group was fed a liquid diet containing $35 \%$ fat, $18 \%$ protein and $47 \%$ carbohydrates provided by Trophic Animal Feed High-tech Co., Ltd. for 8 weeks. Each diet contains the same minerals and vitamins, and all liquid diets were prepared once a day for one day only.

To construct the MAFLD mouse model, animals were fed a Western-style diet (34\% fat, 17\% protein and 49\% carbohydrates, provided by Trophic Animal Feed High-tech Co., Ltd.) and aseptic water for 8 weeks. The MAFLD control group was fed a standard control diet (10\% fat, 17\% protein and $73 \%$ carbohydrate, provided by Trophic Animal Feed High-tech Co., Ltd.) and aseptic water for 8 weeks (Fig. 1).

Sample collection. On the 56th day, the mice were anesthetized by intraperitoneal injection of $1 \%$ pentobarbital sodium (40 mg/kg) and then sacrificed by cervical dislocation. The contents of the small intestine were collected and stored at $-80^{\circ} \mathrm{C}$. Blood samples were harvested from the removed eyeballs and centrifuged to separate plasma $\left(1,500 \mathrm{x} \mathrm{g}, 4^{\circ} \mathrm{C}\right.$, $10 \mathrm{~min}$ ). The plasma was used for analysis of liver marker enzymes. Liver tissue was immediately fixed in $4 \%$ paraformaldehyde and embedded in paraffin.

Biochemical analyses. The plasma lipopolysaccharide (LPS; cat. no. A054-1-1), alanine aminotransferase (ALT; cat. no. C009-2-1), aspartate aminotransferase (AST; cat. no. C010-2-1), triglyceride (TG; cat. no. A110-1-1) and total cholesterol (TC; cat. no. A111-1-1) levels were measured using 


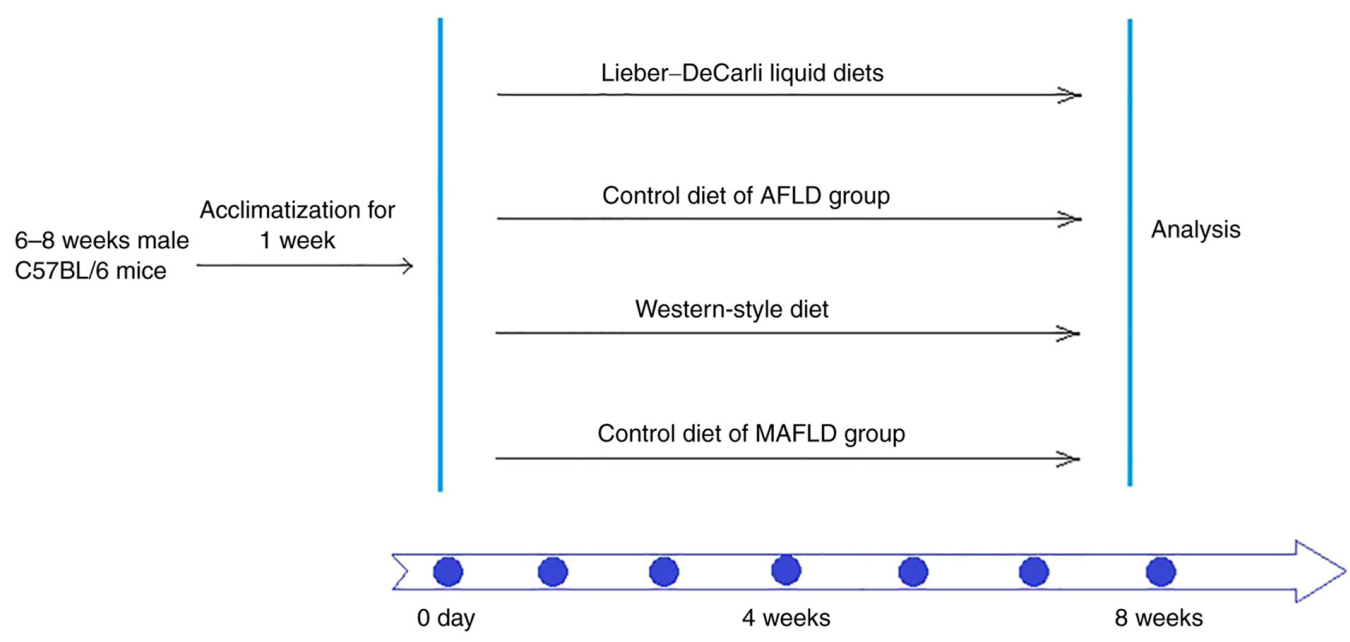

Figure 1. Flow chart of the experiment ( $\mathrm{n}=7$ mice/group). AFLD, alcoholic fatty liver disease; MAFLD, metabolic-associated fatty liver disease.

commercial kits (Nanjing Jiancheng Bioengineering Institute) according to the manufacturer's protocol. Interleukin (IL)-6 (cat. no. H007), IL-1ß (cat. no. H002), IL-10 (cat. no. H009) and tumor necrosis factor (TNF)- $\alpha$ (cat. no. H052) levels were measured by an ELISA kit (Nanjing Jiancheng Bioengineering Institute) as specified by the manufacturer.

Histopathology. The fixed liver tissues were embedded in paraffin, sliced into sections ( $4 \mu \mathrm{m}$ thickness) and stained with hematoxylin and eosin (H\&E). H\&E-stained liver sections were examined under an Olympus microscope. An Olympus digital camera controlled by Olympus Standard software (Olympus Corporation) were used to capture images at original magnifications of $\mathrm{x} 200$.

High-throughput $16 S$ ribosomal (r)RNA amplicon sequencing. The $16 \mathrm{~S}$ rDNA high-throughput sequencing was performed by Novogene using the Ion S5 ${ }^{\mathrm{TM}}$ XL platform (Ion S5 ${ }^{\mathrm{TM}}$ XL Ion 530 Chip; Thermo Fisher Scientific, Inc.). Total genomic DNA from samples was extracted using the cetyltrimethylammonium bromide/SDS method $(23,24)$. The DNA concentration and purity were monitored on $1 \%$ agarose gels using a standard sample, and according to the known concentration of the standard, DNA was diluted to $\sim 1 \mathrm{ng} / \mu \mathrm{l}$ using sterile water. Regions of the 16S rRNA genes (16SV3-V4) were amplified using specific primers (515F-806R) with barcodes. All PCRs were performed in 30- $\mu$ l reaction volumes with $15 \mu \mathrm{l}$ of Phusion ${ }^{\circledR}$ High-Fidelity PCR Master Mix (New England Biolabs), $0.2 \mu \mathrm{M}$ of forward and reverse primers and $10 \mathrm{ng}$ of template DNA. Thermocycling consisted of initial denaturation at $98^{\circ} \mathrm{C}$ for $1 \mathrm{~min}$, followed by 30 cycles of denaturation at $98^{\circ} \mathrm{C}$ for $10 \mathrm{sec}$, annealing at $50^{\circ} \mathrm{C}$ for $30 \mathrm{sec}$ and elongation at $72^{\circ} \mathrm{C}$ for $30 \mathrm{sec}$, with a final extension at $72^{\circ} \mathrm{C}$ for $5 \mathrm{~min}$. The same volume of $1 \mathrm{X}$ loading buffer (containing SYBR green) was mixed with the PCR products and electrophoresis was performed on $2 \%$ agarose gel for detection. PCR products were mixed in equidensity ratios and PCR product mixtures were purified with a GeneJET ${ }^{\mathrm{TM}}$ Gel Extraction kit (Thermo Fisher Scientific, Inc.). Sequencing libraries were generated using the Ion Plus Fragment Library Kit 48 rxns (Thermo Fisher Scientific, Inc.) following the manufacturer's recommendations. The library quality was assessed on a Qubit@2.0 Fluorometer (Thermo Fisher Scientific, Inc.). Finally, the library was sequenced on an IonS5 ${ }^{\mathrm{TM}}$ XL platform and 400/600-bp single-end reads were generated. Single-end reads were assigned to samples based on their unique barcode and truncated by cutting off the barcode and primer sequence. Quality filtering on the raw reads was performed under specific filtering conditions to obtain high-quality clean reads according to the Cutadapt (v1.9.1; http://cutadapt.readthedocs. io/en/stable/) quality control process. The reads were compared with the reference database (Silva database) (25) using the UCHIME algorithm (26) to detect chimera sequences and the chimera sequences were removed (27). Finally, the clean reads were obtained. Sequence analysis was performed using Uparse software (Uparse v7.0.1001) (28). Sequences with $\geq 97 \%$ similarity were assigned to the same operational taxonomic units (OTUs). Representative sequences for each OTU were screened for further annotation. For each representative sequence, the Silva database (25) was used based on the Mothur algorithm to annotate taxonomic information. To study the phylogenetic relationships of different OTUs and the differences of the dominant species in different samples (groups), multiple sequence alignment was performed using MUSCLE software (version 3.8.31) (29). OTU abundance information was normalized using a standard of a sequence number corresponding to the sample with the least sequences. Subsequent analyses of $\alpha$-diversity and $\beta$-diversity were performed based on the normalized output data.

16S rDNA gene analysis. A species accumulation boxplot was used to evaluate whether the sample size was sufficient. A Venn diagram was used to display the similarity and overlap of OTUs in multiple groups. The $\alpha$-diversity indices (Chao1, observed_species) were calculated by using QIIME version 1.7.0 (http://qiime.org/scripts/split_libraries_fastq. html). $\beta$-diversity, which represents a comparison of the microbial community composition and provides a measure of differences between microbial communities, was visualized by nonmetric multidimensional scaling (NMDS), principal coordinates analysis (PCOA) and unweighted pair-group method with arithmetic mean (UPGMA). The relative abundances 
of species with the top 10 abundances in each group at the phylum level are displayed in a histogram. The relative abundance levels of the species with the top 35 abundances in each group at the family and genus level were displayed in a heatmap. To identify the species with a significantly different abundance in the gut microbiota between groups, Student's $\mathrm{t}$-test was used $(\mathrm{P}<0.05)$.

Statistical analysis. All statistical analyses were performed using SPSS version 25.0 (IBM Corp.). Graphs were generated using Prism 8.0 (GraphPad Software, Inc.). Differences in LPS, ALT, AST, TG, TC and inflammatory cytokines were evaluated by one-way ANOVA followed by Tukey's post-hoc test and a significant difference was considered when $\mathrm{P}<0.05$. For the 16S rDNA gene sequencing data of the gut microbiota, the analysis was performed using $\mathrm{R}$ software (version 2.15.3). The analysis of similarities (ANOSIM) test was used to analyze the difference of $\beta$-diversity. The species of gut microbiota with significant difference was identified by Student's t-test. $\mathrm{P}<0.05$ was considered to indicate a statistically significant difference.

\section{Results}

Effects of AFLD and MAFLD on LPS, ALT, AST, TG and TC levels in plasma. To investigate the effects of chronic alcohol feeding and a Western-style diet on the plasma parameters in the liver, the plasma LPS, ALT, AST, TG and TC levels were measured. In comparison with the respective control group, the contents of LPS in the plasma were significantly increased in the AFLD group $(\mathrm{P}<0.01)$ but were not obviously changed in the MAFLD group $(\mathrm{P}>0.05)$. A $153 \%$ increase in the LPS content in plasma was observed in the AFLD group (Fig. 2).

Plasma ALT and AST levels are usually used to evaluate liver injury. In the present study, the plasma ALT and AST levels were significantly increased in the AFLD group and only ALT levels were significantly increased in the MAFLD group, in comparison with the respective control group $(\mathrm{P}<0.05)$. Furthermore, both the AFLD and MAFLD groups exhibited higher levels of TG than their control groups $(\mathrm{P}<0.05)$, but the level of TC increased in the MAFLD group only (Fig. 2). These results suggested that chronic alcohol feeding and a Western-style diet may lead to hypercholesterolemia in mice.

Effects of AFLD and MAFLD on liver inflammatory cytokines and pathology. A previous study indicated that steatosis and inflammation develop as a result of excessive proinflammatory factors in MASH (30). Thus, the cytokines in mouse plasma were measured (Fig. 3). The plasma levels of TNF- $\alpha$ and IL-1 $\beta$ were significantly increased in mice following chronic alcohol feeding, while the change in IL-6 and IL-10 level was not significant. However, the MAFLD group had significantly increased plasma IL-6, IL-1 $\beta$ and TNF- $\alpha$ levels and decreased plasma IL-10 compared with the control.

Histopathological analysis was performed to assess morphological changes (Fig. 4). The pathological morphology of liver sections from the AFLD and MAFLD groups displayed hemorrhagic lesions and an irregular cell arrangement in hepatic parenchyma compared to the control groups. In addition, excessive infiltration of inflammatory cells in liver tissue and lipid vacuoles were observed in hepatocytes from the MAFLD group and Mallory bodies in hepatocytes from the AFLD group compared to those from the control groups.

Differences in microbial richness and diversity between the FLD and control groups. The species accumulation boxplots reached stable values, indicating that the sequencing covered most phylotypes (Fig. 5). Microbial richness and evenness are presented in Fig. 6. The results suggested that 458 of all OTUs accounting for total richness were universal to all samples of the AFLD and AFLD control group, which revealed overlapping data of the 2 groups in a Venn diagram, and 390 OTUs of the AFLD group were distinct from those of the AFLD control group (Fig. 6A). The $\alpha$-diversity of the AFLD group was altered compared with that of the control group and the Chaol and observed_species index were increased in the AFLD group. In conclusion, the microbial richness and evenness were increased in the AFLD group compared with those in the AFLD control group (Fig. 6C and D).

A total of 254 OTUs of the MAFLD group were distinct from those of the MAFLD control group, demonstrating marked differences in the MAFLD group (Fig. 6B). Regarding $\alpha$-diversity, the Chaol and observed_species index in the MAFLD group was decreased compared with that in the MAFLD control group (Fig. 6E and F). In general, the microbiota richness was decreased in the MAFLD group compared with that in the MAFLD control group.

Differences in microbial community structure between the FLD and FLD control groups. Data on the $\beta$-diversity are presented in Fig. 7. The NMDS, PCoA and hierarchical clustering analysis by UPGMA of weighted UniFrac distances indicated obvious clustering between the AFLD group and the AFLD control group (NMDS, stress <0.2; Fig. 7A, B and E). The results indicated that the gut microbiota community of the AFLD group was significantly different from that of the control group, indicating that chronic alcohol feeding influenced the gut microbiota. The ANOSIM test, which was used to determine whether the difference between groups was significantly higher compared with that within groups suggested that the observed cluster patterns were significant $(\mathrm{R}=0.4295, \mathrm{P}=0.002)$, indicating that the difference between groups was greater than that within groups $(R>0$ indicates that the difference between groups is greater than that within groups).

Results of the analysis of the microbial community structure between the MAFLD and MAFLD control groups by weighted UniFrac distances NMDS, PCoA and hierarchical clustering analysis by UPGMA are provided in Fig. 7C, D and F, respectively. The NMDS (stress <0.2), PCoA and hierarchical clustering analysis displayed the clusters of the two groups and suggested that the MAFLD group had an altered gut microbiota structure. The ANOSIM test indicated that the difference between groups was greater than that within groups $(\mathrm{R}=0.4441, \mathrm{P}=0.001)$.

Shifts in gut microbiota induced by FLD. In the AFLD group, the species with the highest abundances at the phylum level were Firmicutes, Actinobacteria, Proteobacteria and Bacteroidetes 

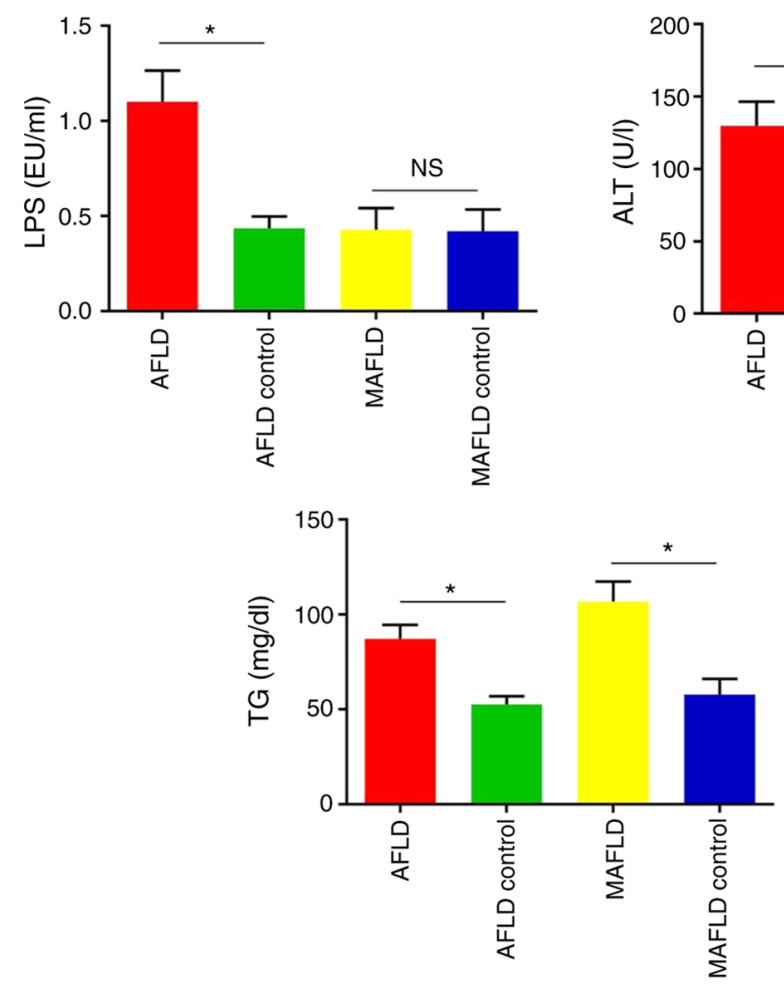
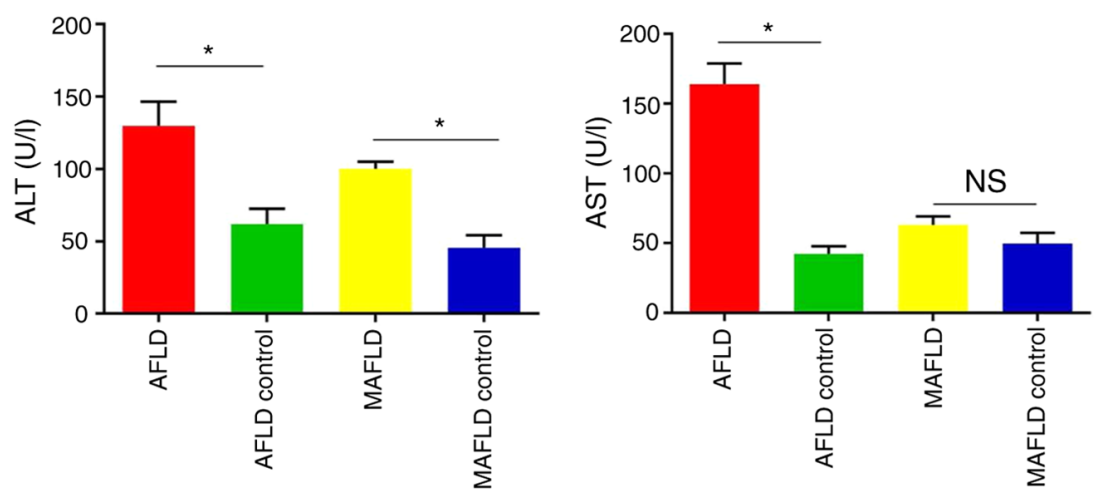

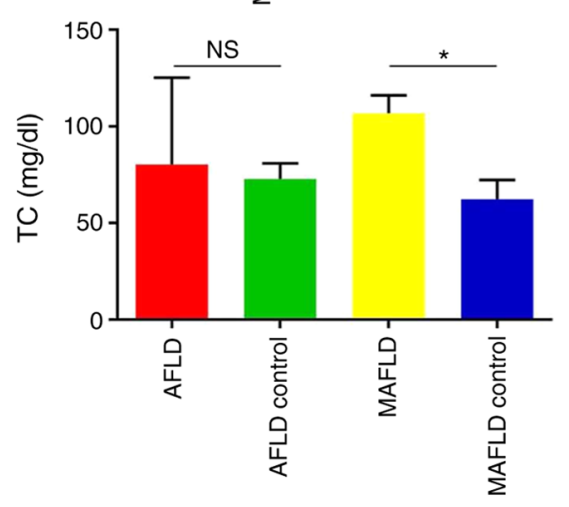

Figure 2. Impact of the Lieber-DeCarli liquid diet (AFLD group) and Western-style diet (MAFLD group) on liver function. Plasma levels of liver function serological markers, including LPS, ALT, AST, TG and TC. The Lieber-DeCarli liquid diet (AFLD group) significantly increased the levels of LPS, ALT, AST and TG in plasma and the Western-style diet (MAFLD group) significantly increased the levels of ALT, TG, and TC in the plasma of mice. Values are expressed as the mean \pm standard deviation $(\mathrm{n}=5)$. ${ }^{*} \mathrm{P}<0.05$. NS, no significant difference; AFLD, alcoholic fatty liver disease; MAFLD, metabolic-associated fatty liver disease; ALT, alanine aminotransferase; AST, aspartate aminotransferase; TG, triglyceride; TC, total cholesterol; LPS, lipopolysaccharide.
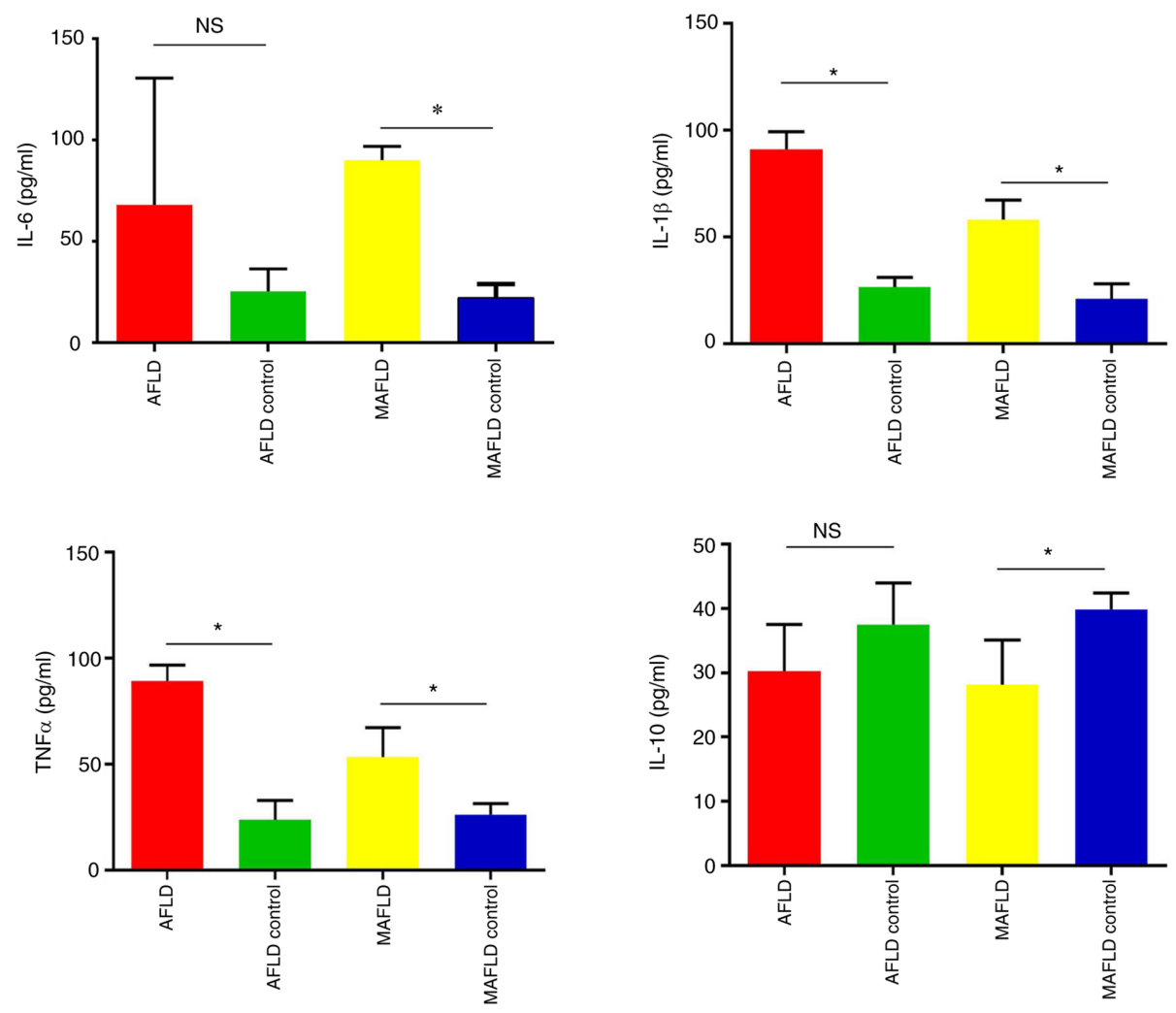

Figure 3. Impact of the Lieber-DeCarli liquid diet (AFLD group) and Western-style diet (MAFLD group) on plasma inflammatory cytokines. Plasma inflammatory cytokine levels of IL-6, IL-1 $\beta$, TNF- $\alpha$ and IL-10. The Lieber-DeCarli liquid diet (AFLD group) significantly increased the levels of IL-1 $\beta$ and TNF- $\alpha$ in plasma, while the Western-style diet (MAFLD group) led to an increase in IL-6, IL-1 $\beta$ and TNF- $\alpha$ and a decrease in IL-10 in the plasma of mice. Values are expressed as the mean \pm standard deviation $(\mathrm{n}=5)$. ${ }^{*} \mathrm{P}<0.05$. NS, no significant difference; AFLD, alcoholic fatty liver disease; MAFLD, metabolic-associated fatty liver disease; IL, interleukin; TNF, tumor necrosis factor. 

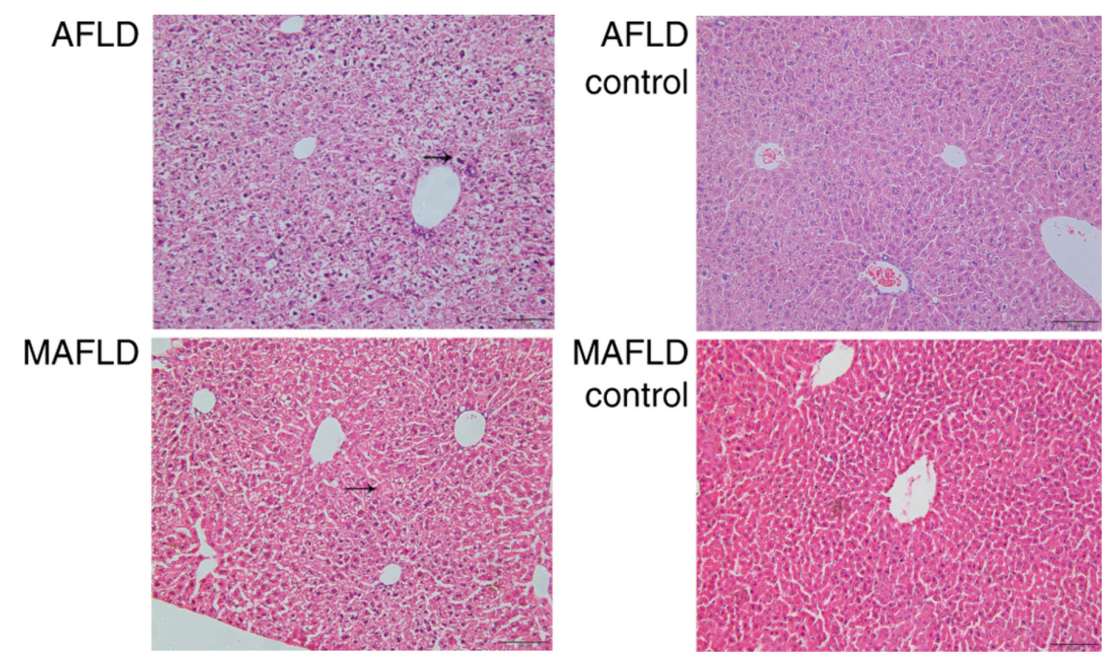

Figure 4. Pathological changes in the liver in mice. Representative histopathological images of H\&E-stained hepatic tissues from mice fed a Lieber-DeCarli liquid diet (AFLD group) and Western-style diet (MAFLD group). H\&E staining revealed an increase in infiltration of inflammatory cells and lipid vacuoles (indicated by an arrow in the MAFLD group) in liver tissue from the MAFLD group and Mallory bodies (indicated by an arrow in the AFLD group) in hepatocytes from the AFLD group compared to those from the control groups. AFLD, alcoholic fatty liver disease; MAFLD, metabolic-associated fatty liver disease. Scale bars, $50 \mu \mathrm{m}$.

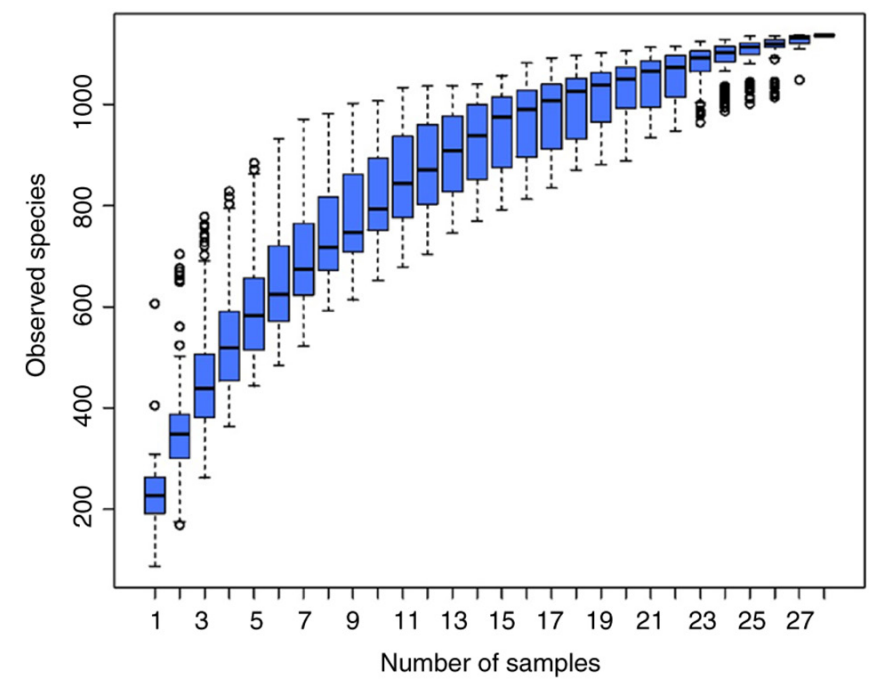

Figure 5. Species accumulation boxplot. The species accumulation boxplots reached stable values, indicating that the sequencing covered most phylotypes. The values are expressed as operational taxonomic units.

(Fig. 8A). However, no significant difference was observed at the phylum level between the AFLD and AFLD control groups. In the MAFLD group, the species with the highest abundances at the phylum level were Firmicutes, Actinobacteria, Proteobacteria and Verrucomicrobia (Fig. 8B).

To compare the gut microbiota between the two groups with significant differences, a t-test was performed (Fig. 9A and B). Comparison between the AFLD and AFLD control groups at the family level indicated that the main bacterium in the AFLD group was Enterococcaceae $(\mathrm{P}=0.027)$. At the genus level, the main bacteria in the AFLD group were Enterococcus $(\mathrm{P}=0.027)$ and Streptococcus $(\mathrm{P}=0.021)$. However, no gut microbiota were significantly different in the AFLD control group compared with those in the AFLD group at the phylum level.
Comparisons of the gut microbiota between the MAFLD and the MAFLD control group are provided in Fig. 9C-E. A significant increase in the proportion of Firmicutes $(\mathrm{P}=0.01)$ and a decrease in the proportion of Actinobacteria $(\mathrm{P}=0.006)$ in the MAFLD group were identified at the phylum level. At the family level, the MAFLD group mainly consisted of Lachnospiraceae $(\mathrm{P}=0.005)$ and the MAFLD control group mainly consisted of Bifidobacteriaceae $(\mathrm{P}=0.019)$, Lactobacillaceae $(\mathrm{P}=0.033)$ and Atopobiaceae $(\mathrm{P}=0.029)$. At the genus level, the MAFLD group included Gordonibacter $(\mathrm{P}=0.046)$, Erysipelatoclostridium $(\mathrm{P}=0.011)$ and Streptococcus $(\mathrm{P}=0.004)$, and the main bacteria in the MAFLD control group were Bifidobacterium $(\mathrm{P}=0.019)$ and Lactobacillus $(\mathrm{P}=0.033)$.

\section{Discussion}

The present study indicated that AFLD and MAFLD caused changes, including increases in the plasma LPS, ALT, AST, TG, IL- $1 \beta$ and TNF- $\alpha$ in the AFLD group and increases in the plasma ALT, TG, TC, IL-6, IL-1 $\beta$ and TNF- $\alpha$ and a decrease in the plasma IL-10 in the MAFLD group. In addition, macroscopic evaluation of the severity of liver disease indicated that compared with mice in the control groups, the mice in the chronic alcohol-induced AFLD group and the Western-style diet-induced MAFLD group exhibited obvious characteristics of fatty liver. Finally, different compositions of the gut microbiota in the AFLD and MAFLD groups were observed. The results indicated that in comparison to the AFLD control group, Enterococcaceae were the most abundant bacteria at the family level and Enterococcus and Streptococcus were the most abundant bacteria at the genus level in the AFLD group. In the MAFLD group, Lachnospiraceae was the most abundant at the family level, with increases in Erysipelatoclostridium, Gordonibacter and Streptococcus at the genus level and a decrease in the genus Bifidobacterium. 
A
AFLD

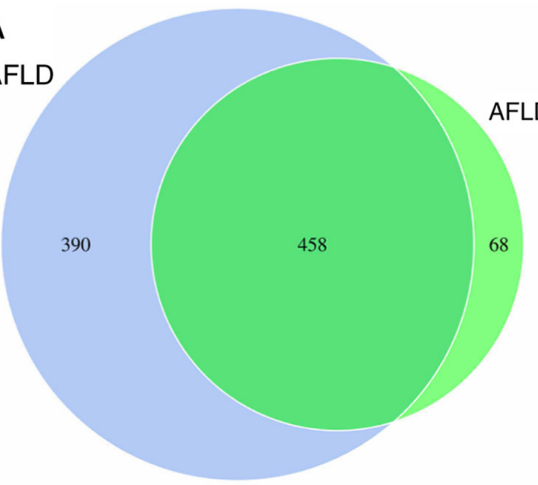

C

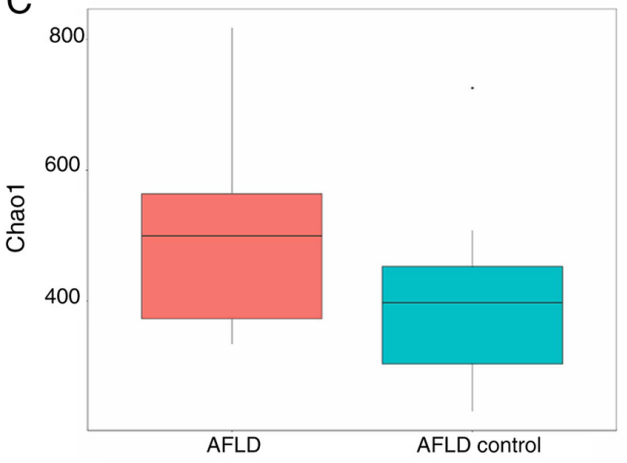

E

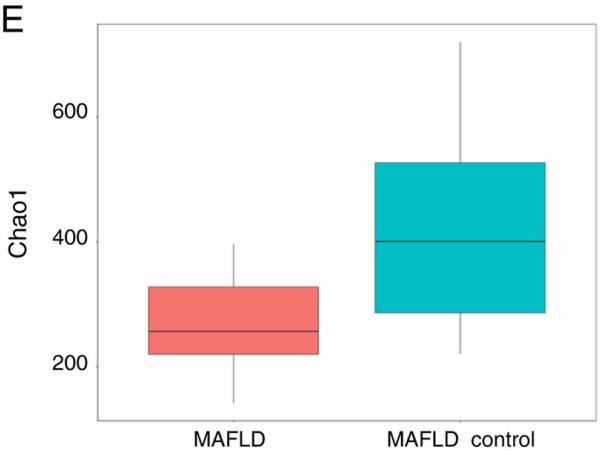

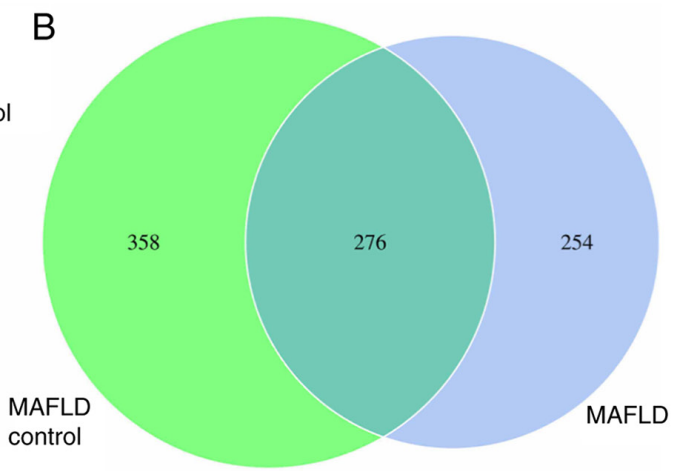
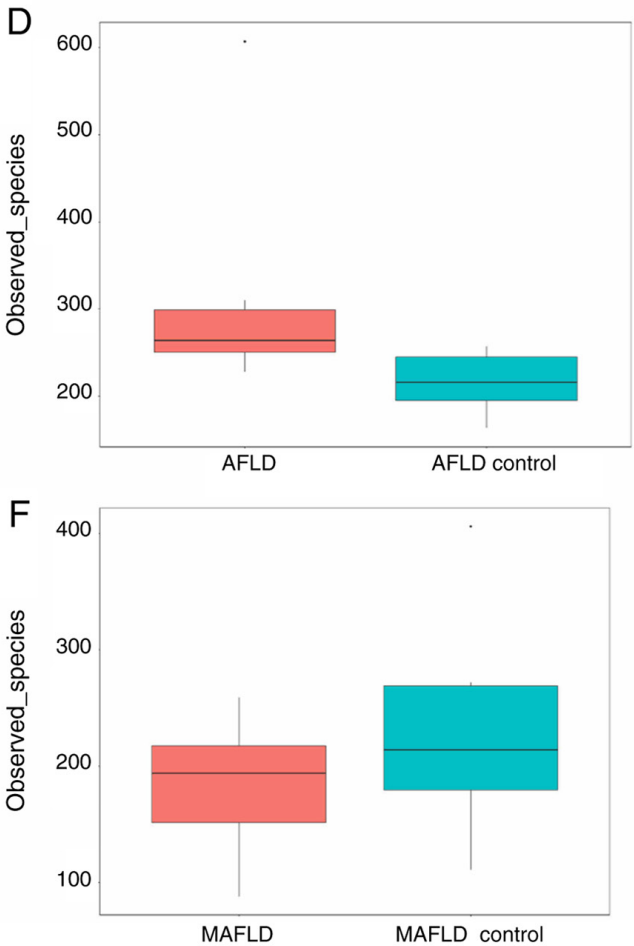

Figure 6. Bacterial species richness in the two groups. The Lieber-DeCarli liquid diet (AFLD group) led to an increase, while the Western-style diet (MAFLD group) led to a decrease in bacterial species richness. (A) A Venn diagram was used to indicate the similarity and overlap of OTU between the AFLD and control groups: The number of OTUs in common between the AFLD group and AFLD control group was 458 and the number of unique OTUs of the AFLD group was 390, compared with 36 in the AFLD control group. (B) Venn diagram for the MAFLD and control groups: The number of OTUs in common between the MAFLD group and MAFLD control group was 276, and the number of unique OTUs of the MAFLD group was 254 compared with 358 in the MAFLD control group. (C) The $\alpha$-diversity index Chaol and (D) observed_species: A measure of species richness and evenness from chronic alcohol feeding-induced AFLD and the control group. The AFLD group had a higher bacterial diversity than the control group, suggesting that AFLD increased the bacterial species richness. (E and F) In the MAFLD group, (E) Chaol and (F) observed_species were lower than those of the control group, suggesting that the bacterial species richness and evenness were lower in the MAFLD group. Taking all of the above together, the bacterial species richness was increased in the AFLD group and decreased in the MAFLD group as compared with that in their respective control groups. AFLD, alcoholic fatty liver disease; MAFLD, metabolic-associated fatty liver disease; OTU, operational taxonomic units.

It was previously indicated that the concentration of LPS is associated with chronic liver inflammation (31). In the present study, a significant increase in the LPS level in the plasma of the AFLD group was observed. Therefore, LPS may have an important role in the chronic inflammation of AFLD. The results also revealed that the plasma liver functional markers were significantly higher in the AFLD (ALT and AST) and MAFLD (ALT) groups, consistent with previous studies $(32,33)$.

Inflammatory cytokines have been reported to be important contributing factors to liver diseases (34). In certain patients, FLD may develop into a stage of steatohepatitis $(1,2)$. Therefore, steatohepatitis is an important characteristic of FLD. IL-6 is a proinflammatory cytokine that contributes to the progression of chronic inflammatory proliferative diseases (35). Jorge et al (36) reported that individuals with higher morphological severity of MAFLD exhibited higher IL- 6 and TNF- $\alpha$ expression. Elevated plasma IL- 6 was also associated with increased severity and mortality in patients with alcoholic hepatitis (37). It has been reported that IL-6 pathways may be selectively inhibited and therapeutically exploited for the treatment of liver pathologies (38). Bird et al (39) revealed that the elevations of TNF- $\alpha$ in patients with alcoholic hepatitis were most marked in severe cases, suggesting that TNF has a role in the pathogenesis of this condition. IL-1 $\beta$, which is a member of the IL-1 family, has also been indicated to mediate 

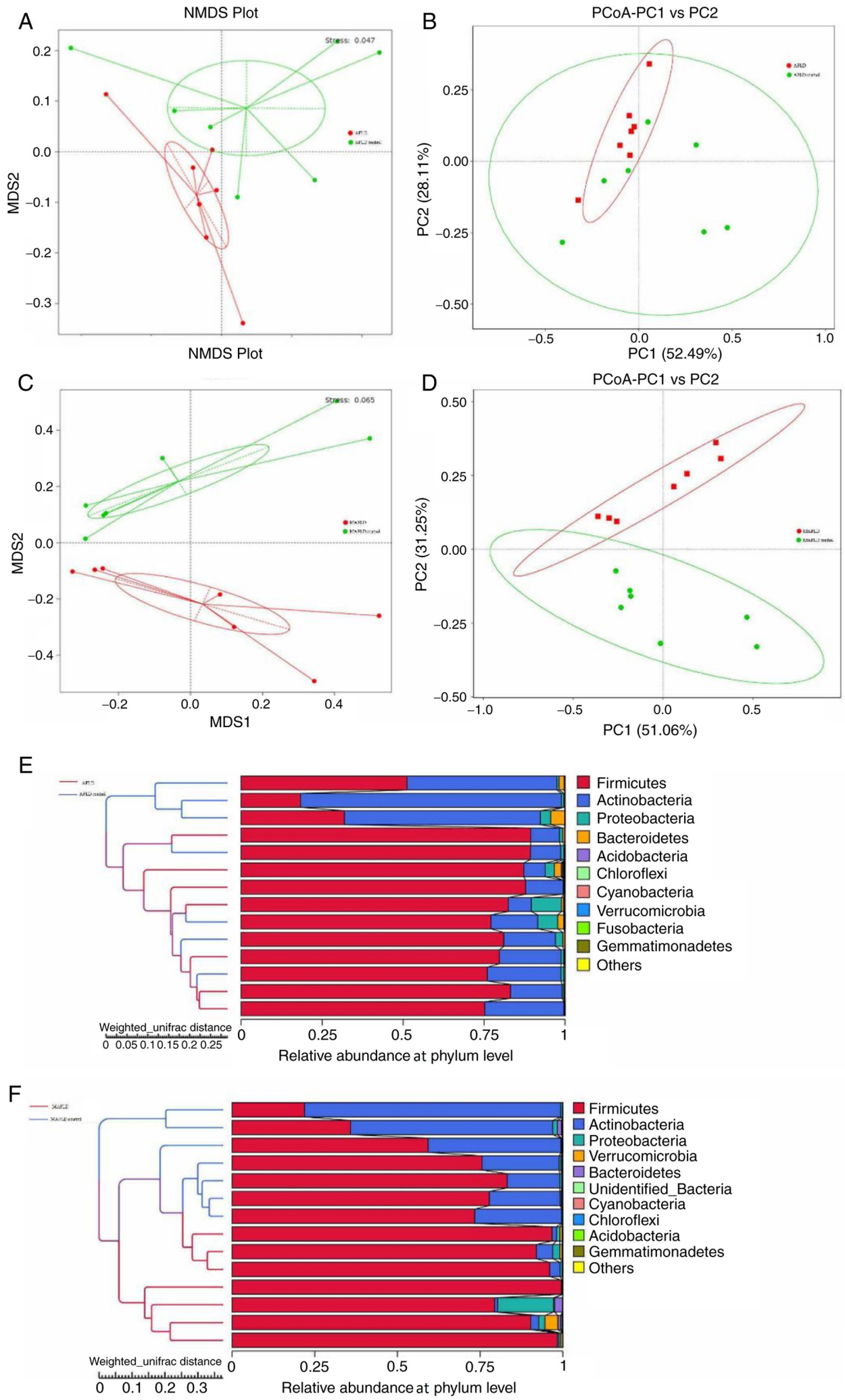

Figure 7. Altered structure of the gut microbiota by the Lieber-DeCarli liquid diet (AFLD group) and Western-style diet (MAFLD group). (A) The $\beta$-diversity analysis plot was generated by NMDS for the AFLD and control groups. (B) Plots presented were generated by PCoA for the AFLD and control groups. (C) Plots presented were generated by NMDS for the MAFLD and control groups. (D) Plots were generated by PCoA for the MAFLD and control groups. (E and F) Hierarchical clustering analysis based on weighted UniFrac distances for (E) the AFLD group and AFLD control group and (F) MAFLD group and MAFLD control group. On the left, the unweighted pair-group method with arithmetic mean cluster tree structure between groups is provided and on the right, the relative abundance distribution map of species at the phylum level is displayed for each sample. The number of branches represents the phylogenetic distance, with shorter branches indicating a closer evolutionary relationship. In the NMDS analysis in A and C, when the stress is $<0.2$, the NMDS may accurately reflect the difference between samples. In the PCoA analysis in B and D, the abscissa represents the first principal component and the percentage represents the contribution value of the first principal component to the sample difference; the ordinate represents the second principal component and the percentage represents the contribution value of the second principal component to the sample difference; each point in the graph represents a sample and the samples of the same group are represented by the same color. AFLD, alcoholic fatty liver disease; MAFLD, metabolic-associated fatty liver disease; NMDS, nonmetric multidimensional scaling; PCoA, principal coordinates analysis. 

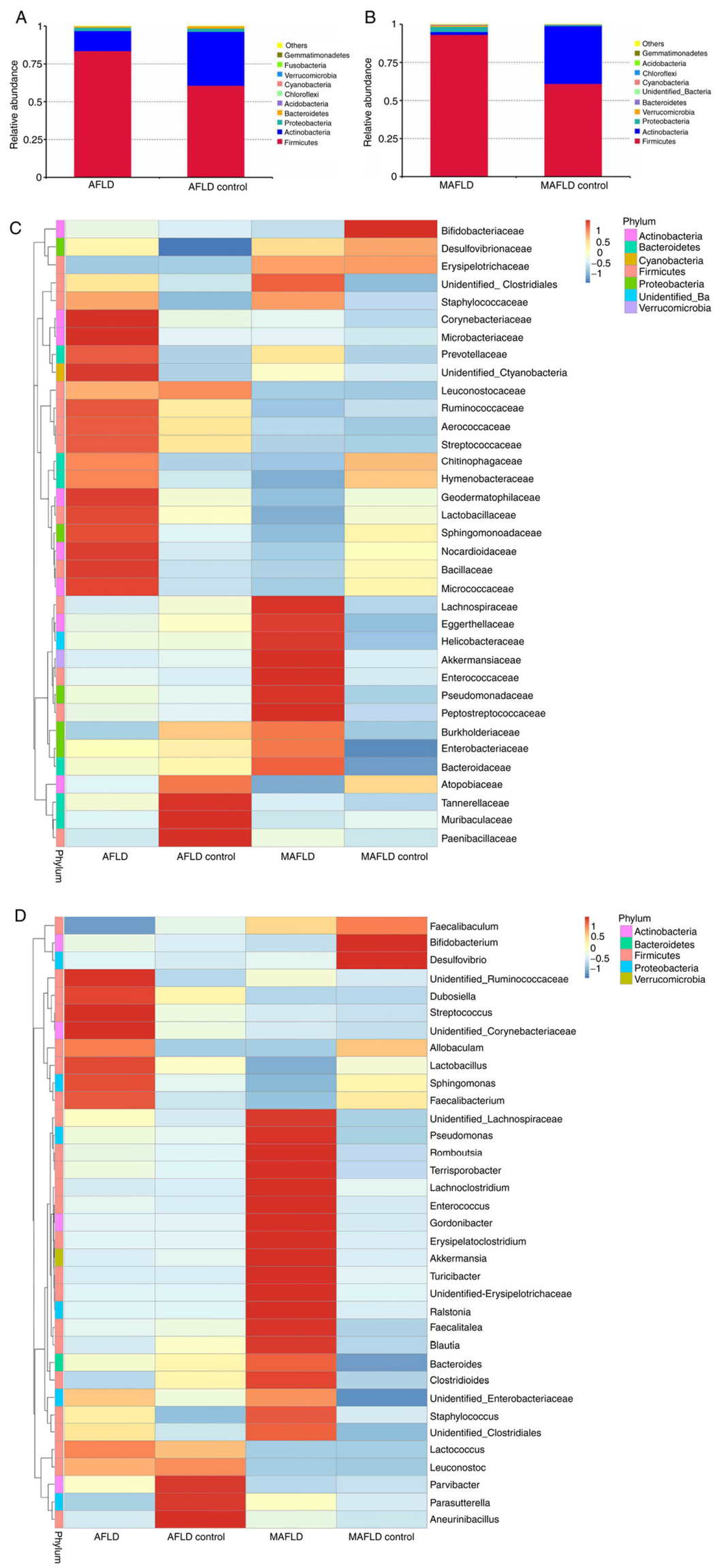

Figure 8. Relative abundances of species at different levels for each group. (A) Relative abundances of species with the highest 10 abundances at the phylum level in the AFLD and control groups. (B) Relative abundances of species with the highest 10 abundances at the phylum level in the MAFLD and control groups. (C and D) Community heatmap according to the species records and abundance information of all groups at (C) the family level and (D) the genus level. Using the maximum ranking method, the groups with the top 35 abundances (the average abundance of all samples in the group) were selected and clustered from the species level according to their abundance information in each group. AFLD, alcoholic fatty liver disease; MAFLD, metabolic-associated fatty liver disease. 
A

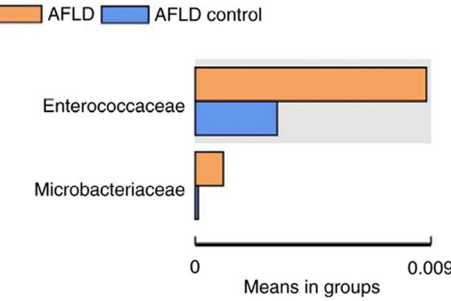

$B \square$ AFLD $\square$ AFLD control

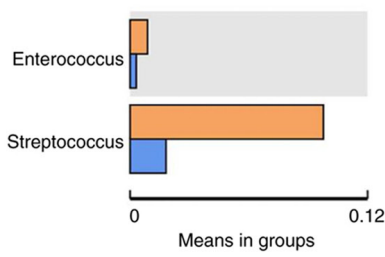

C

$\square$ MAFLD $\square$ MAFLD control

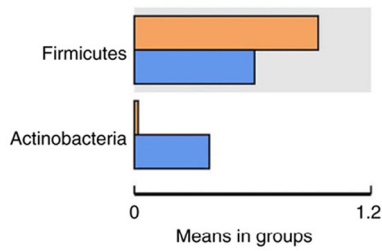

$\mathrm{D} \square$ MAFLD $\square$ MAFLD control

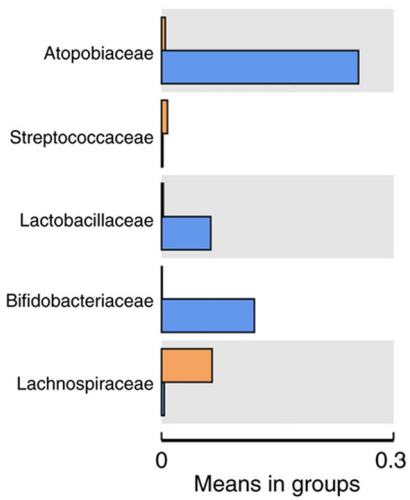

E

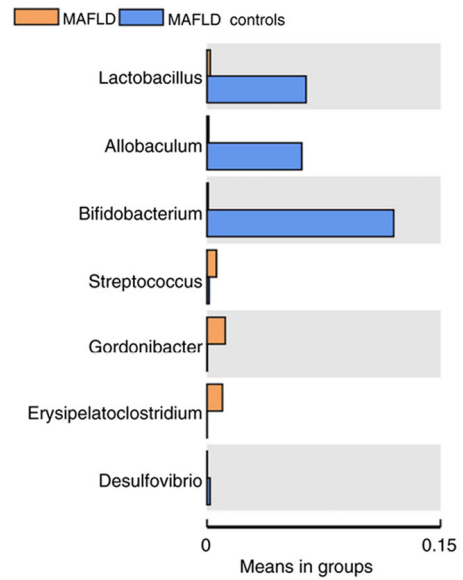

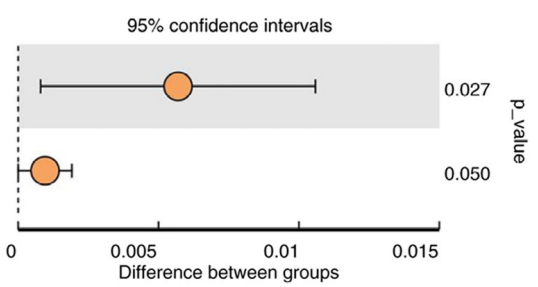
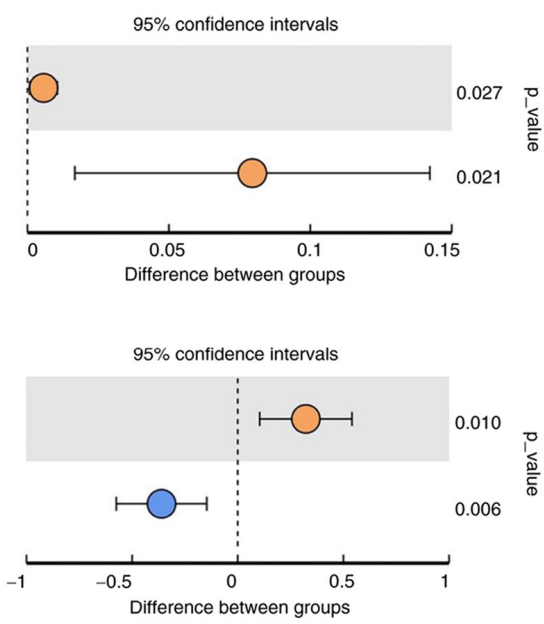

$95 \%$ confidence intervals

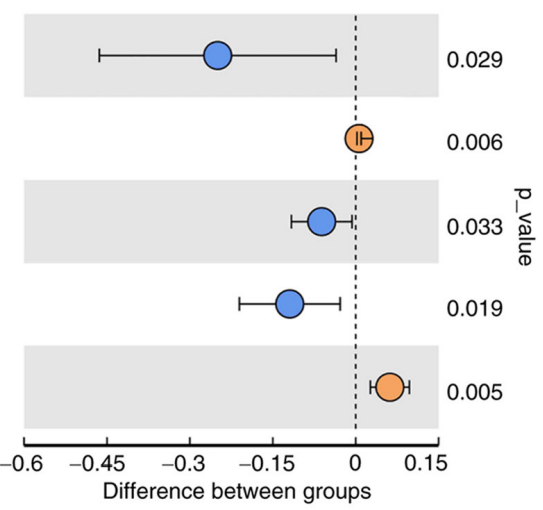

$95 \%$ confidence intervals

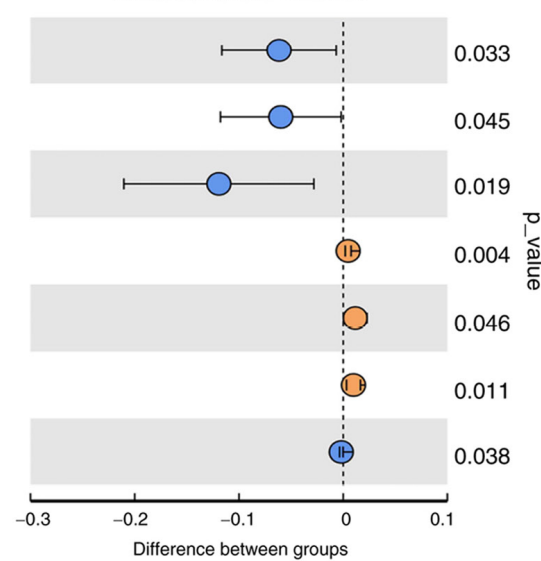

Figure 9. Discrepancy of the gut microbiota composition between groups. (A and B) Species with significant differences between the AFLD and control groups were analyzed at (A) the family level and (B) the genus level. (C-E) Species with significant differences between the MAFLD and control groups at (C) the phylum level, (D) the family level and (E) at the genus level. A significant discrepancy was defined as $\mathrm{P}<0.05$ according to the t-test. In the panel on the left, the abundance of different species is compared between groups and each bar represents the average value of species with significant differences in abundance between groups. The figure on the right indicates the confidence of the difference between groups. The leftmost end of the line connecting each circle represents the lower limit of the $95 \%$ confidence interval of the mean difference and the rightmost end of the line connecting each circle represents the upper limit of the 95\% confidence interval of the mean difference. The center of the circle represents the difference of the mean. The rightmost end of the display result is the intergroup significance test P-value corresponding to the different species. AFLD, alcoholic fatty liver disease; MAFLD, metabolic-associated fatty liver disease. 


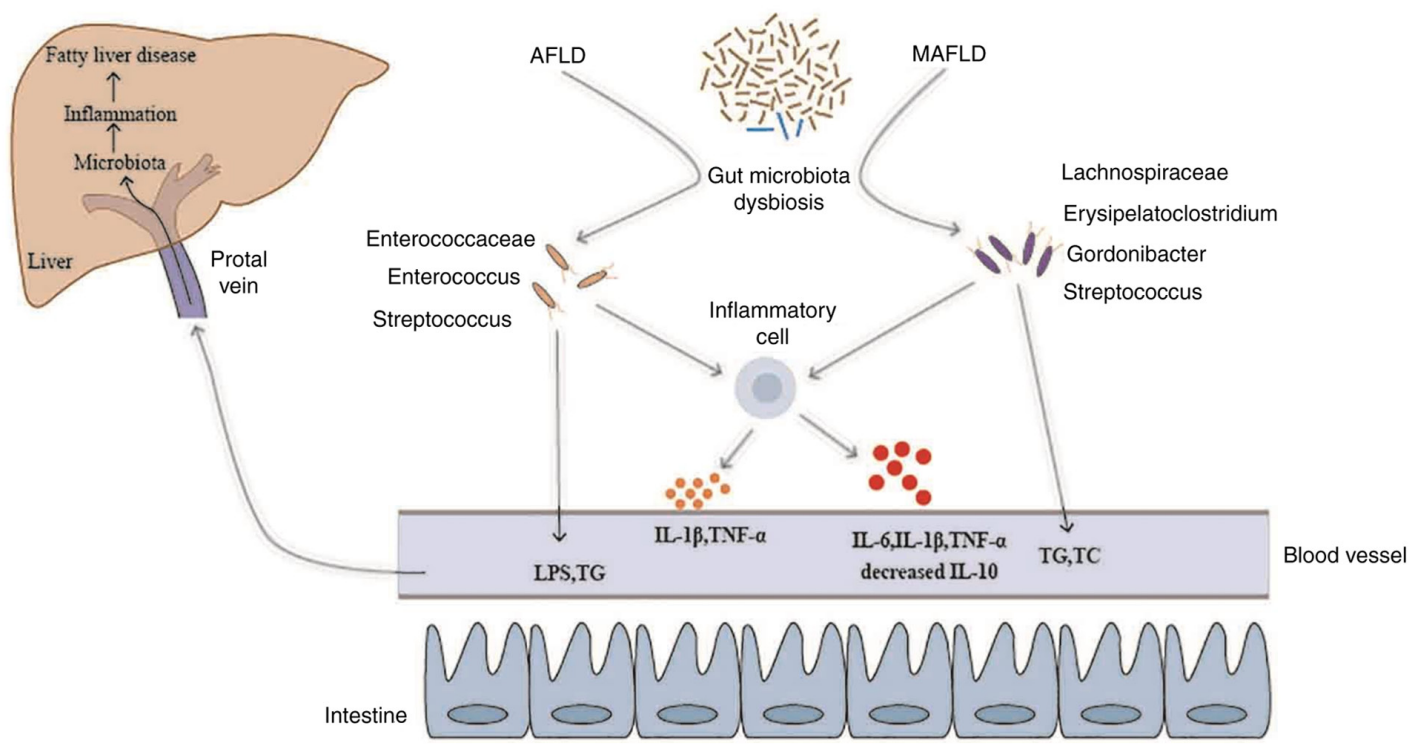

Figure 10. Schematic representation of the proposed process of microbial translocation during FLD. After the onset of gut dysbiosis, inflammatory cells of the intestinal wall are activated and produce inflammatory cytokines to increase intestinal permeability. The gut microbiota and their products may cross the mucosal barrier to reach the liver through the portal circulation. Gut microbiota and their products cause hepatic inflammation and liver disease. AFLD, alcoholic fatty liver disease; MAFLD, metabolic-associated fatty liver disease; TG, triglyceride; TC, total cholesterol; LPS, lipopolysaccharide; IL, interleukin; $\mathrm{TNF}$, tumor necrosis factor.

different aspects in both AFLD and MAFLD (40). In addition, growing evidence indicated that increased production of IL-10 may help protect against AFLD and MAFLD by counteracting the effects of proinflammatory factors $(41,42)$. Consistent with these studies, the present results also indicated increased levels of IL- $1 \beta$ and TNF- $\alpha$ in the AFLD group and increased levels of IL-6, IL-1 $\beta$ and TNF- $\alpha$, as well as a decreased levels of IL-10, in the MAFLD group. However, the plasma levels of IL-6 and IL-10 were not significantly different in the AFLD group compared with those in the AFLD control group, which may be due to the different animals and induction methods used. Finally, the effects in the AFLD and MAFLD groups were further confirmed by conventional histological assessment of the animals' livers.

In the present study, it was observed that the gut microbial composition of mice with AFLD was obviously changed compared with that of the control group, which may be clinically significant. The results indicated increased $\alpha$-diversity (microbial richness and evenness) in the AFLD group. The increase in the $\alpha$-diversity index indicated that chronic alcohol feeding led to gut disorders, which increased the diversity of microbiota in the gut and increased the number of pathogens, as demonstrated by $\mathrm{t}$-test analysis. In addition, the $\beta$-diversity suggested a comparatively heterogeneous community between the MAFLD mice and control mice. The ANOSIM test suggested that the composition of the gut microbiota between the two groups was significantly different compared with the respective control group.

The increased abundance levels of the family Enterococcaceae and genera Enterococcus and Streptococcus were the characteristic changes in the gut microbiota in the AFLD group. The increase in Enterococcaceae fit well with the results of a previous study, which suggested that Enterococcaceae was a predominant contributor to the development and progression of primary liver cancer (43). Zhang et al (44) also indicated higher levels of Enterobacteriaceae in cirrhotic rats than in healthy rats. Consistent with the present results, Duan et al (45) reported an increase in the bacterium Enterococcus in the alcoholic hepatitis group compared with that in a healthy control group. In a recent study, it was suggested that Enterococcus gallinarum has the ability to trigger autoimmune responses by translocating to the liver and other systemic tissues (46). In addition, bacteriophages, which are able to eliminate Enterococcus faecalis, have been reported to be capable of preventing AFLD (47). The results of shifts in Enterococcus are in agreement with the study of Llorente et al (48), who reported that gastric acid suppression may promote alcoholic liver disease by inducing overgrowth of intestinal Enterococcus, further confirming the pathogenic role of Enterococcus in AFLD. Furthermore, Nakayama et al (49) revealed that alcohol consumption promoted the intestinal translocation of Streptococcus suis infections. The level of Streptococcus was significantly increased in the present study, in line with the findings of Posteraro et al (50). In conclusion, the results suggested that chronic alcohol feeding may change the gut microbiota of mice through the production of harmful bacteria (Enterobacteriaceae, Streptococcus and Enterococcus).

Changes in the gut microbiota community have been reported in MAFLD models (51). The present study determined that the MAFLD group displayed a lower overall $\alpha$-diversity. Furthermore, based on the OTU information, the t-test was used to assess the different gut microbiota compositions at different levels. At the phylum level, there was a predominance of Firmicutes in the MAFLD group, which was in agreement with the results of previous studies, demonstrating that Firmicutes are the main phyla in MAFLD (52). However, these results differed from those of another study on patients with MASH, which indicated a decrease of Firmicutes in the 
MASH group (53). The reason for this phenomenon remains elusive, but several factors (mainly animal and diet) may be implicated. Certain characteristic changes in bacteria, such as higher proportions of the family Lachnospiraceae and lower proportions of the family Lactobacillaceae, have been detected in patients with MAFLD (54). The level of Lachnospiraceae has also been indicated to be significantly increased in patients with MASH compared with that in controls (55). Similar to the current observations, the abundance of opportunistic bacteria (genus Erysipelatoclostridium) has been reported to be decreased in mice treated with antiaging agents (56). Smith-Brown et al (57) further demonstrated that Erysipelatoclostridium was also associated with dairy-based food intake in 2- to 3-year-old Australian children. In addition, the levels of Gordonibacter have been reported to be increased in Chinese patients with multiple system atrophy compared with controls (58). As members of Firmicutes, the genus Streptococcus, which was significantly higher in patients with MAFLD in comparison with individuals without the disease, has been identified as a possible marker (59). In another study, high-density energy diets have also been reported to induce microbiota dysbiosis within a week of introducing the diet and induce marked hepatic lipidosis after 4 weeks (13). In the MAFLD control group, the different dysbiosis of gut microbiota indicated that a high carbohydrate diet may also influence the composition of gut microbiota in a different way, which was similar to a previous study (60). These prior studies, along with the present results, suggest that specific aberrations in the normal gut microbiota may be associated with the development of MAFLD.

Healthy gut levels of Bifidobacterium are well known to modulate the immune response and protect the intestinal barrier (61). On the one hand, Bifidobacterium may produce short-chain fatty acids, which are energy sources for intestinal epithelial cells and are also crucial for gut immune homeostasis (62). On the other hand, Bifidobacterium may ameliorate MAFLD through Gpr109a, which is a short chain fatty acid receptor recognized and activated by butyric acid in adipocytes, hepatocytes and colon cells, and the commensal metabolite butyrate $(63,64)$. The results of the present study were consistent with those of the above-mentioned previous reports, indicating that a Western-style diet-induced MAFLD in mice affect the composition of gut microbiota by increasing harmful bacteria (family Lachnospiraceae; genus Gordonibacter, Erysipelatoclostridium and Streptococcus) and decreasing beneficial bacteria (Bifidobacterium).

Accumulating evidence has indicated that the gut microbiota was associated with disease severity-activated inflammatory cells in adults with MAFLD (65). In patients with primary liver cancer, Streptococcus has been reported to be positively correlated with the level of AST $(\mathrm{P}<0.05)$ and Bifidobacterium was negatively correlated with the levels of ALT and AST $(\mathrm{P}<0.05)(43)$. Oo et al (66) reported that the probiotic FK-23 (heat-treated Enterococcus faecalis strain FK-23), which was given at 2,700 mg per day via the oral route, was able to reduce the levels of plasma ALT and AST in hepatitis C virus-positive patients. In another study, the results indicated that deficiencies of IL-10, IL-10R $\alpha$ and IL-10R $\beta$ may result in dysbiosis of the caecal microbiota in mice, characterized by expanded populations of opportunistic bacteria of the families Enterococcaceae and Enterobacteriaceae (67). In patients with immune deficiency syndrome, the amount of Enterococcus faecalis was positively correlated with the contents of TNF- $\alpha$ and IL- 6 and the CD4 ${ }^{+}$ T-lymphocyte count (68). In patients with liver cirrhosis, the level of Enterococcus has also been indicated to be positively correlated with AST levels $(\mathrm{P}<0.05)$ and Bifidobacterium was negatively correlated with AST $(\mathrm{P}<0.05)$ (69). Furthermore, the metabolites of Bifidobacterium infantis have been reported to protect immature human enterocytes from IL- $1 \beta$-induced inflammation (70). All of these reports are consistent with the present results indicating that inflammatory cells of the intestine wall are activated and produce inflammatory cytokines to increase intestinal permeability. The gut microbiota and their products may cross the mucosal barrier to reach the liver through the portal circulation resulting in hepatic inflammation (Fig. 10).

However, there is a limitation to the present study that requires to be addressed. Only samples from C57BL/6 mice were assessed in the present study. Therefore, human stool samples from patients with MAFLD/AFLD could be examined in the future. Although the present study does not provide evidence of a direct causal relationship between these bacteria and FLD, the present results provide preliminary insight into changes in the gut microbiota in AFLD and MAFLD compared with the respective control group. In the future, the development of techniques such as metabolomics and metagenomics may reveal the function of the gut microbiota and will improve the understanding of the structure and function of the gut microbiota.

In conclusion, the present study reported a chronic inflammatory response and gut microbiota dysbiosis in the AFLD and MAFLD mouse models. Both the AFLD and MAFLD groups had increased levels of ALT, TG, IL-1 $\beta$ and TNF- $\alpha$. However, LPS, AST increased significantly in the AFLD group only, TC, IL-6 increased in the MAFLD group only and IL-10 decreased significantly in the MAFLD group only. Furthermore, the AFLD group presented with increased richness of the gut microbiota, while the MAFLD group exhibited decreased richness. The characteristic changes in the gut microbiota of the AFLD group were increased Enterobacteriaceae, Streptococcus and Enterococcus. The changes in the gut microbiota in the MAFLD group included increases in Lachnospiraceae, Erysipelatoclostridium, Gordonibacter and Streptococcus and a decrease in Bifidobacterium. Although the direct association of the gut microbiota with FLD remains elusive, the present study may provide an experimental basis for future studies on the interaction between the microbiota and FLD. Further studies are necessary to explore the role of specific gut microbiota in the development of FLD.

\section{Acknowledgements}

Not applicable.

\section{Funding}

The present study was supported by the Innovative Talent Support Program of the Institution of Higher Learning in 
Liaoning Province (grant no. 2018-478), the support project of the Shenyang Science Plan (grant no. 20-205-4-094) and the Innovative Talents of Science and Technology Support Programs of Young and Middle-aged People of Shenyang (grant no. RC170446).

\section{Availability of data and materials}

The datasets used and/or analyzed during the present study are available from the corresponding author on reasonable request.

\section{Authors' contributions}

KK performed the laboratory experiments, analyzed the data and wrote the manuscript. LXS, YS and BC performed parts of the experiments. DP, YLL and MJS conceived the study. $\mathrm{KK}$ and $\mathrm{BC}$ confirmed the authenticity of the raw data. All authors read and approved the final manuscript.

\section{Ethics approval and consent to participate}

All procedures were in strict accordance with the National Institutes of Health guidelines and were approved by the Animal Research Committee of China Medical University (Shenyang, China; no. 2019061).

\section{Patient consent for publication}

Not applicable.

\section{Competing interests}

The authors declare that they have no competing interests.

\section{References}

1. Szabo G, Kamath PS, Shah VH, Thursz M and Mathurin P; EASL-AASLD Joint Meeting: Alcohol-related liver disease: Areas of consensus, unmet needs and opportunities for further study. Hepatology 69: 2271-2283, 2019.

2. Teli MR, Day CP, Burt AD, Bennett MK and James OF: Determinants of progression to cirrhosis or fibrosis in pure alcoholic fatty liver. Lancet 346: 987-990, 1995.

3. Araújo AR, Rosso N, Bedogni G, Tiribelli C and Bellentani S: Global epidemiology of non-alcoholic fatty liver disease/non-alcoholic steatohepatitis: What we need in the future. Liver Int 38 (Suppl 1): S47-S51, 2018.

4. Levene AP and Goldin RD: The epidemiology, pathogenesis and histopathology of fatty liver disease. Histopathology 61: 141-152, 2012.

5. Yu Y, Cai J, She Z and Li H: Insights into the epidemiology, pathogenesis, and therapeutics of nonalcoholic fatty liver diseases. Adv Science (Weinh) 6: 1801585, 2019.

6. Peredo-Lovillo A, Romero-Luna HE and Jiménez-Fernández M: Health promoting microbial metabolites produced by gut microbiota after prebiotics metabolism. Food Res Int 136: 109473, 2020.

7. Willers M, Ulas T, Völlger L, Vogl T, Heinemann AS, Pirr S, Pagel J, Fehlhaber B, Halle O, Schöning J, et al: S100A8 and S100A9 are important for postnatal development of gut microbiota and immune system in mice and infants. Gastroenterology 159: 2130-2145.e5, 2020

8. Ruff WE, Greiling TM and Kriegel MA: Host-microbiota interactions in immune-mediated diseases. Nat Rev Microbiol 18: 521-538, 2020.

9. Fan Y and Pedersen O: Gut microbiota in human metabolic health and disease. Nat Rev Microbiol 19: 55-71, 2021.

10. Jiang L and Schnabl B: Gut microbiota in liver disease: What do we know and what do we not know? Physiology (Bethesda) 35 261-274, 2020.
11. Lang S, Fairfied B, Gao B, Duan Y, Zhang X, Fouts DE and Schnabl B: Changes in the fecal bacterial microbiota associated with disease severity in alcoholic hepatitis patients. Gut Microbes 12: 1785251, 2020.

12. Ferrere G, Wrzosek L, Cailleux F, Turpin W, Puchois V, Spatz M, Ciocan D, Rainteau D, Humbert L, Hugot C, et al: Fecal microbiota manipulation prevents dysbiosis and alcohol-induced liver injury in mice. J Hepatol 66: 806-815, 2017.

13. Minaya DM, Turlej A, Joshi A, Nagy T, Weinstein N, DiLorenzo P, Hajnal A and Czaja K: Consumption of a high energy density diet triggers microbiota dysbiosis, hepatic lipidosis, and microglia activation in the nucleus of the solitary tract in rats. Nutr Diabetes 10: 20, 2020.

14. Shen F, Zheng RD, Sun XQ, Ding WJ, Wang XY and Fan JG: Gut microbiota dysbiosis in patients with non-alcoholic fatty liver disease. Hepatobiliary Pancreat Dis Int 16: 375-381, 2017.

15. Kim HN, Joo EJ, Cheong HS, Kim Y, Kim HL, Shin H, Chang Y and Ryu S: Gut microbiota and risk of persistent nonalcoholic fatty liver diseases. J Clin Med 8: 1089, 2019.

16. Martín R, Chain F, Miquel S, Motta JP, Vergnolle N, Sokol H and Langella P: Using murine colitis models to analyze probiotics-host interactions. FEMS Microbiol Rev 41 (Supp 1): S49-S70, 2017.

17. Liu L, Li P, Liu Y and Zhang Y: Efficacy of probiotics and synbiotics in patients with nonalcoholic fatty liver disease: A meta-analysis. Dig Dis Sci 64: 3402-3412, 2019.

18. Kirpich IA, Solovieva NV, Leikhter SN, Shidakova NA, Lebedeva OV, Sidorov PI, Bazhukova TA, Soloviev AG, Barve SS, McClain CJ and Cave M: Probiotics restore bowel flora and improve liver enzymes in human alcohol-induced liver injury: A pilot study. Alcohol 42: 675-682, 2008.

19. Lamas-Paz A, Hao F, Nelson LJ, Vázquez MT, Canals S, Gómez Del Moral M, Martínez-Naves E, Nevzorova YA and Cubero FJ: Alcoholic liver disease: Utility of animal models. World J Gastroenterol 24: 5063-5075, 2018.

20. Zhong F, Zhou X, Xu J and Gao L: Rodent models of nonalcoholic fatty liver disease. Digestion 101: 522-535, 2020.

21. Yoneyama N, Crabbe JC, Ford MM, Murillo A and Finn DA: Voluntary ethanol consumption in 22 inbred mouse strains. Alcohol 42:149-160, 2008.

22. Denucci SM, Tong M, Longato L, Lawton M, Setshedi M, Carlson RI, Wands JR and de la Monte SM: Rat strain differences in susceptibility to alcohol-induced chronic liver injury and hepatic insulin resistance. Gastroenterol Res Pract 2010: 312790, 2010.

23. Lutz KA, Wang W, Zdepski A and Michael TP: Isolation and analysis of high quality nuclear DNA with reduced organellar DNA for plant genome sequencing and resequencing. BMC Biotechnol 11: 54, 2011.

24. Zhang L and Wang S: Bacterial community diversity on in-shell walnut surfaces from six representative provinces in China. Sci Rep 7: 10054, 2017.

25. Quast C, Pruesse E, Yilmaz P, Gerken J, Schweer T, Yarza P, Peplies J and Glöckner FO: The SILVA ribosomal RNA gene database project: Improved data processing and web-based tools. Nucleic Acids Res 41 (Database issue): D590-D596, 2013.

26. Edgar RC, Haas BJ, Clemente JC, Quince C and Knight R: UCHIME improves sensitivity and speed of chimera detection. Bioinformatics 27: 2194-2200, 2011.

27. Haas BJ, Gevers D, Earl AM, Feldgarden M, Ward DV, Giannoukos G, Ciulla D, Tabbaa D, Highlander SK, Sodergren E, et al: Chimeric 16S rRNA sequence formation and detection in Sanger and 454-pyrosequenced PCR amplicons. Genome Res 21: 494-504, 2011.

28. Edgar RC: UPARSE: Highly accurate OTU sequences from microbial amplicon reads. Nat Methods 10: 996-998, 2013.

29. Edgar RC: MUSCLE: Multiple sequence alignment with high accuracy and high throughput. Nucleic Acids Res 32: 1792-1797, 2004.

30. Hadinia A, Doustimotlagh AH, Goodarzi HR, Arya A and Jafarinia M: Circulating levels of pro-inflammatory cytokines in patients with nonalcoholic fatty liver disease and non-alcoholic steatohepatitis. Iran J Immunol 16: 327-333, 2019.

31. Carpino G, Del Ben M, Pastori D, Carnevale R, Baratta F, Overi D, Francis H, Cardinale V, Onori P, Safarikia S, et al: Increased liver localization of lipopolysaccharides in human and experimental NAFLD. Hepatology 72: 470-485, 2020.

32. Yan AW, Fouts DE, Brandl J, Stärkel P, Torralba M, Schott E, Tsukamoto H, Nelson KE, Brenner DA and Schnabl B: Enteric dysbiosis associated with a mouse model of alcoholic liver disease. Hepatology 53: 96-105, 2011. 
33. Jung F, Lippmann T, Brandt A, Jin CJ, Engstler AJ and Baumann A: Moderate consumption of fermented alcoholic beverages diminishes diet-induced non-alcoholic fatty liver disease through mechanisms involving hepatic adiponectin signaling in mice. Eur J Nutr 59: 787-799, 2020.

34. Xiong DD, Zhang M, Li N, Gai JF, Mao L and Li M: Mediation of inflammation, obesity and fatty liver disease by advanced glycation endoproducts. Eur Rev Med Pharmacol Sci 21: 5172-5178, 2017.

35. Ishihara $\mathrm{K}$ and Hirano T: IL-6 in autoimmune disease and chronic inflammatory proliferative disease. Cytokine Growth Factor Rev 13: 357-368, 2002.

36. Jorge ASB, Andrade JMO, Paraíso AF, Jorge G, Silveira CM, de Souza LR, Santos EP, Guimaraes A, Santos S and De-Paula A: Body mass index and the visceral adipose tissue expression of IL-6 and TNF-alpha are associated with the morphological severity of non-alcoholic fatty liver disease in individuals with class III obesity. Obes Res Clin Pract 12 (Suppl 2): S1-S8, 2018.

37. Sheron N, Bird G, Goka J, Alexander G and Williams R: Elevated plasma interleukin- 6 and increased severity and mortality in alcoholic hepatitis. Clin Exp Immunol 84: 449-453, 1991.

38. Schmidt-Arras D and Rose-John S: IL-6 pathway in the liver: From physiopathology to therapy. J Hepatol 64: 1403-1415, 2016.

39. Bird GL, Sheron N, Goka AK, Alexander GJ and Williams RS : Increased plasma tumor necrosis factor in severe alcoholic hepatitis. Ann Intern Med 112: 917-920, 1990.

40. Tilg H, Moschen AR and Szabo G: Interleukin-1 and inflammasomes in alcoholic liver disease/acute alcoholic hepatitis and nonalcoholic fatty liver disease/nonalcoholic steatohepatitis. Hepatology 64: 955-965, 2016.

41. Hill DB, D'Souza NB, Lee EY, Burikhanov R, Deaciuc IV and de Villiers WJ: A role for interleukin-10 in alcohol-induced liver sensitization to bacterial lipopolysaccharide. Alcohol Clin Exp Res 26: 74-82, 2002

42. Paredes-Turrubiarte G, González-Chávez A, Pérez-Tamayo R, Salazar-Vázquez BY, Hernández VS, Garibay-Nieto N, Fragoso JM and Escobedo G: Severity of non-alcoholic fatty liver disease is associated with high systemic levels of tumor necrosis factor $\alpha$ and low serum interleukin 10 in morbidly obese patients. Clin Exp Med 16: 193-202, 2016.

43. Zhang L, Wu YN, Chen T, Ren CH, Li X and Liu GX: Relationship between intestinal microbial dysbiosis and primary liver cancer. Hepatobiliary Pancreat Dis Int 18: 149-157, 2019.

44. Zhang W, Gu Y, Chen Y, Deng H, Chen L, Chen S, Zhang G and Gao Z: Intestinal flora imbalance results in altered bacterial translocation and liver function in rats with experimental cirrhosis. Eur J Gastroenterol Hepatol 22: 1481-1486, 2010.

45. Duan Y, Llorente C, Lang S, Brandl K, Chu H, Jiang L, White RC, Clarke TH, Nguyen K, Torralba M, et al: Bacteriophage targeting of gut bacterium attenuates alcoholic liver disease. Nature 575: 505-511, 2019.

46. Manfredo Vieira S, Hiltensperger M, Kumar V, Zegarra-Ruiz D, Dehner C, Khan N, Costa FRC, Tiniakou E, Greiling T, Ruff W, et al: Translocation of a gut pathobiont drives autoimmunity in mice and humans. Science 359: 1156-1161, 2018.

47. Çolakoğlu M, Xue J and Trajkovski M: Bacteriophage prevents alcoholic liver disease. Cell 180: 218-220, 2020.

48. Llorente C, Jepsen P, Inamine T, Wang L, Bluemel S, Wang HJ, Loomba R, Bajaj JS, Schubert ML, Sikaroodi M, et al: Gastric acid suppression promotes alcoholic liver disease by inducing overgrowth of intestinal Enterococcus. Nat Commun 8: 837, 2017.

49. Nakayama T, Takeuchi D, Matsumura T, Akeda Y, Fujinaga Y and Oishi K: Alcohol consumption promotes the intestinal translocation of Streptococcus suis infections. Microb Pathog 65: 14-20, 2013

50. Posteraro B, Paroni Sterbini F, Petito V, Rocca S, Cubeddu T, Graziani C, Arena V, Vassallo GA, Mosoni C, Lopetuso L, et al Liver injury, endotoxemia, and their relationship to intestinal microbiota composition in alcohol-preferring rats. Alcohol Clin Exp Res 42: 2313-2325, 2018.

51. Zhang DY, Zhu L, Liu HN, Tseng YJ, Weng SQ, Liu TT, Dong L and Shen XZ: The protective effect and mechanism of the FXR agonist obeticholic acid via targeting gut microbiota in non-alcoholic fatty liver disease. Drug Des Devel Ther 13: 2249-2270, 2019.

52. Yang Y, Yang F, Huang M, Wu H, Yang C, Zhang X, Yang L, Chen G, Li S, Wang Q, et al: Fatty liver and alteration of the gut microbiome induced by diallyl disulfide. Int J Mol Med 44: 1908-1920, 2019.

53. Zhu L, Baker SS, Gill C, Liu W, Alkhouri R, Baker RD and Gill SR: Characterization of gut microbiomes in nonalcoholic steatohepatitis (NASH) patients: A connection between endogenous alcohol and NASH. Hepatology 57: 601-609, 2013.
54. Raman M, Ahmed I, Gillevet PM, Probert CS, Ratcliffe NM, Smith S, Greenwood R, Sikaroodi M, Lam V, Crotty P, et al: Fecal microbiome and volatile organic compound metabolome in obese humans with nonalcoholic fatty liver disease. Clin Gastroenterol Hepatol 11: 868-875, 2013.

55. Boursier J, Mueller O, Barret M, Machado M, Fizanne L, Araujo-Perez F, Guy CD, Seed PC, Rawls JF, David LA, et al: The severity of nonalcoholic fatty liver disease is associated with gut dysbiosis and shift in the metabolic function of the gut microbiota. Hepatology 63: 764-775, 2016.

56. Luo D, Chen K, Li J, Fang Z, Pang H, Yin Y, Rong X and Guo J: Gut microbiota combined with metabolomics reveals the metabolic profile of the normal aging process and the anti-aging effect of FuFang Zhenshu TiaoZhi(FTZ) in mice. Biomed Pharmacother 121: 109550, 2020.

57. Smith-Brown P, Morrison M, Krause L and Davies PS: Dairy and plant based food intakes are associated with altered faecal microbiota in 2 to 3 year old Australian children. Sci Rep 6: 32385, 2016.

58. Du J, Huang P, Qian Y, Yang X, Cui S, Lin Y, Gao C, Zhang P, He Y, Xiao Q and Chen S: Fecal and blood microbial 16s rRNA gene alterations in Chinese patients with multiple system atrophy and its subtypes. J Parkinsons Dis 9: 711-721, 2019.

59. Nistal E, Sáenz de Miera LE, Ballesteros Pomar M, Sánchez-Campos S, García-Mediavilla MV, Álvarez-Cuenllas B, Linares P, Olcoz JL, Arias-Loste MT, García-Lobo JM, et al: An altered fecal microbiota profile in patients with non-alcoholic fatty liver disease (NAFLD) associated with obesity. Rev Esp Enferm Dig 111: 275-282, 2019.

60. Sen T, Cawthon CR, Ihde BT, Hajnal A, DiLorenzo PM, de La Serre CB and Czaja K: Diet-driven microbiota dysbiosis is associated with vagal remodeling and obesity. Physiol Behav 173: 305-317, 2017.

61. Yano JM, Yu K, Donaldson GP, Shastri GG, Ann P, Ma L, Nagler CR, Ismagilov RF, Mazmanian SK and Hsiao EY: Indigenous bacteria from the gut microbiota regulate host serotonin biosynthesis. Cell 161: 264-276, 2015.

62. Horiuchi H, Kamikado K, Aoki R, Suganuma N, Nishijima T, Nakatani A and Kimura I: Bifidobacterium animalis subsp. lactis GCL2505 modulates host energy metabolism via the short-chain fatty acid receptor GPR43. Sci Rep 10: 4158, 2020.

63. Liang Y, Lin C, Zhang Y, Deng Y, Liu C and Yang Q: Probiotic mixture of Lactobacillus and Bifidobacterium alleviates systemic adiposity and inflammation in non-alcoholic fatty liver disease rats through Gpr109a and the commensal metabolite butyrate. Inflammopharmacology 26: 1051-1055, 2018.

64. Koh A, De Vadder F, Kovatcheva-Datchary P and Bäckhed F: From dietary fiber to host physiology: Short-chain fatty acids as key bacterial metabolites. Cell 165: 1332-1345, 2016.

65. Schwenger KJP, Chen L, Chelliah A, Da Silva HE, Teterina A, Comelli EM, Taibi A, Arendt BM, Fischer S and Allard JP: Markers of activated inflammatory cells are associated with disease severity and intestinal microbiota in adults with non-alcoholic fatty liver disease. Int J Mol Med 42: 2229-2237, 2018.

66. Oo KM, Lwin AA, Kyaw YY, Tun WM, Fukada K, Goshima A, Shimada T and Okada S: Safety and long-term effect of the probiotic FK-23 in patients with hepatitis $\mathrm{C}$ virus infection. Biosci Microbiota Food Health 35: 123-128, 2016.

67. Duque-Correa MA, Karp NA, McCarthy C, Forman S, Goulding D, Sankaranarayanan G, Jenkins TP, Reid AJ, Cambridge EL, Ballesteros Reviriego C, et al: Exclusive dependence of IL-10Ra signalling on intestinal microbiota homeostasis and control of whipworm infection. PLoS Pathog 15: e1007265, 2019.

68. Lu J, Ma SS, Zhang WY and Duan JP: Changes in peripheral blood inflammatory factors (TNF- $\alpha$ and IL-6) and intestinal flora in AIDS and HIV-positive individuals. J Zhejiang Univ Sci B 20: 793-802, 2019.

69. Mou H, Yang F, Zhou J and Bao C: Correlation of liver function with intestinal flora, vitamin deficiency and IL-17A in patients with liver cirrhosis. Exp Ther Med 16: 4082-4088, 2018.

70. Guo S, Guo Y, Ergun A, Lu L, Walker WA and Ganguli K: Secreted metabolites of Bifidobacterium infantis and lactobacillus acidophilus protect immature human enterocytes from IL-1 $\beta$-induced inflammation: A transcription profiling analysis. PLoS One 10: e124549, 2015.

This work is licensed under a Creative Commons Attribution-NonCommercial-NoDerivatives 4.0 International (CC BY-NC-ND 4.0) License. 